\title{
WATER -JET CUTTING - A PRECISE METHOD FOR MANUFACTURING PROCESS
}

\author{
Sarvesh Talele \\ Department of Mechanical Engineering \\ Terna Engineering College, Nerul, India \\ Gauresh Rane \\ Department of Mechanical Engineering \\ Terna Engineering College, Nerul, India
}

\begin{abstract}
This paper discusses the review of the waterjet cutting technique used in various industries. This technique is widely known for precision and accuracy while cutting operations. The paper describes the chronological pattern of how the waterjet cutting process is executed. The process ultimately begins with the types of materials it can cut. For example, materials such as Alloys, Steels, Composite materials, Plastic, Rubber and etc are being cut with the resource of the waterjet process. The materials are cut with great precision and accuracy by this process. The actual cutting operation from waterjet machining operations are carried out with the support of abrasive materials. Abrasive materials have the tendency to cut materials water is to provide appropriate pressure i.e. the exact pressure required to cut respective materials is provided by water. The second stage of the paper describes the necessary conditions for the nozzle and orifice selection criterion, along with wear specifications of nozzle and orifice. The subsequent description of how waterjet machining works, the paper reviews the precision and quality of the equipment used while machining. This stage embarks a reason to select a waterjet machining process as the most accurate and effective process while performing, with limited disadvantages and numerous advantages. Thereafter comparing the waterjet machining process with laser and plasma cutting techniques used in industries. This paper represents a comprehensive and meticulous review of the abrasive waterjet cutting process.
\end{abstract}

Keywords - WaterJet Cutting, Nozzle, Orifice, Pump, Laser Cutting, Plasma Cutting.

\section{INTRODUCTION}

Abrasive waterjet machining process occurs to be the most effective process for machining operations. The process at initial stages used in shape cutting, linear cutting of sheets and plates, even in casting for a wide range of materials [5] Wang J. et. al. (2007). However, when technology evolved, now the

\author{
Aishwarya Dalvi \\ Department of Mechanical Engineering \\ Terna Engineering College, Nerul, India \\ Janhavi Nawar \\ Department of Mechanical Engineering \\ Terna Engineering College, Nerul, India
}

process is used for numerous operations in machining purposes such as deburring, polishing, cutting, turning and milling operations. The abrasive used while performing cutting operations is the key factor for accuracy. Since, the actual cutting is obtained with the help of abrasive material water is to provide efficient amount of pressure for cutting operations. Abrasive materials used in waterjet machining are as follows garnet, silica sand, aluminium oxide, silicon carbide, etc. [1] khan et. al. (2007). Abrasive waterjet machining generates high-pressure water which is then passed through a sapphire, this process produces a high-velocity jet that passes through a mixing chamber and strikes at the target material [3] Xu et. al. (2006). This process creates a partial vacuum that triggers the abrasive particles which are fed through the hopper. Hence there is momentum transfer from the jet to the abrasive particles which occurs in a narrow mixing tube, which is called a nozzle [2] Nanduri et. al (2000). This high-pressure water and mixing it with abrasive generates a higher amount of jet energy which makes machining operations feasible.

On the other hand, this operation can be performed on various categories of materials. The materials on which the operations are possible are mentioned in figure 1.

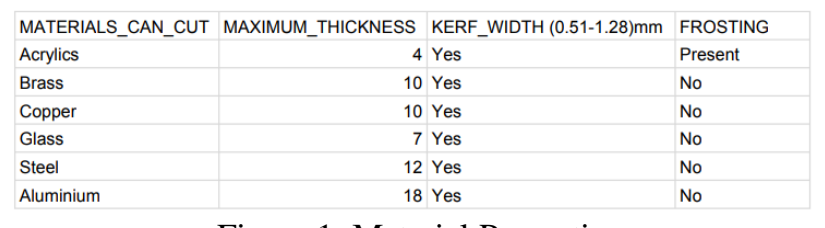

Figure 1: Material Properties

Figure 1 characterizes material as maximum thickness it can cut, frosting on top, and kerf width from $0.51 \mathrm{~mm}-1.28$ $\mathrm{mm}$. Subsequently understanding the material classification, the succeeding step is the selection of nozzle and orifice which is then assembled with the waterjet pump. The selection of nozzle and orifice becomes a crucial role to make operation more precise. Hence design and material selection for nozzle matter extensively. The materials used for the manufacturing of 


\section{International Journal of Engineering Applied Sciences and Technology, 2020 \\ Vol. 4, Issue 11, ISSN No. 2455-2143, Pages 471-473 \\ Published Online March 2020 in IJEAST (http://www.ijeast.com)}

nozzles are silicon carbide, tungsten carbide, boron carbide, tungsten carbide cobalt and also composite carbide. The most widely used material is tungsten carbide to reduce the nozzle wear rate and enhance the nozzle's life. The wear characteristics are explained in more detail in the following paper.

Subsequent selection of nozzle and analyzing design, the vital aspect of waterjet machining is a pump selection criterion. The concept of pressure intensification or amplification which elicits desired output water pressure is applied in an Intensifier. When a certain amount of pressure is applied at one end of the cylinder then the pressure at the other end will be the same, if the diameter of both the ends is identical. Hence, increasing the required amount of pressure from an intensifier, we have to change the inlet and outlet diameter of a cylinder. For effective transmission of pressure the ratio of 1:20 for the surface area is followed. The ratio is defined as the outlet diameter to the inlet diameter of a cylinder. The ratio is fixed for every intensifier to increase the efficiency of waterjet machining. But there should be a restriction to the eminent amount of pressure from the pump, to maintain the same pressure throughout the machining operation. This restriction is generated by the orifice. Pressure is generally maintained until orifice diameter exceeds the water output pump limits. Typically, most of the intensifiers produce 50 - 60 strokes per minute at complete capacity.

Abrasive waterjet machine is known for the precision and accuracy of operations performed. Kerf angle is a significant aspect of the accurate cutting operation, the less is the kerf angle the most precise the design it manufactures. The precision also depends on the nozzle height from the workpiece with appropriate speed. Ultimately the precision, accuracy, and cost are compared with other cutting methods such as Laser Cutting and Plasma Cutting.

\section{IMPORTANT CHARACTERISTICS OF WATERJET MACHINING}

\section{A. Nozzle and Orifice}

The parameters to obtain the desired design of nozzle and orifice. In general, the orifice diameter should be thrice that of the abrasive nozzle for perfect cutting operation [11]. This reduces the part tolerance or kerf width while cutting operations improving cutting efficiency. Figure 1 and Figure 2 represent the orifice (inches) - water flow (GPM) characteristics and abrasive nozzle (inches) - abrasive flow (lbs/min) respectively.

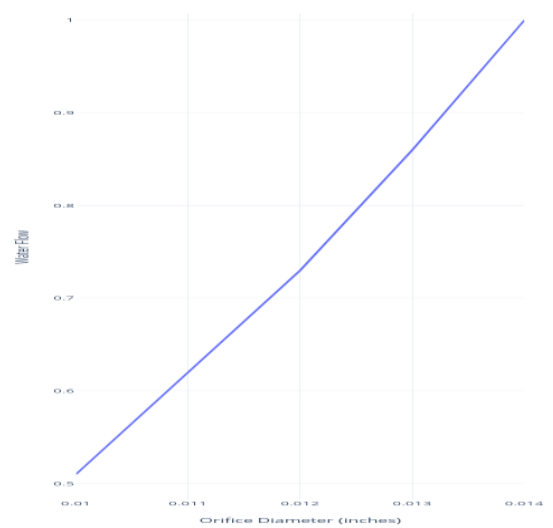

Figure 2 : Orifice - Water flow

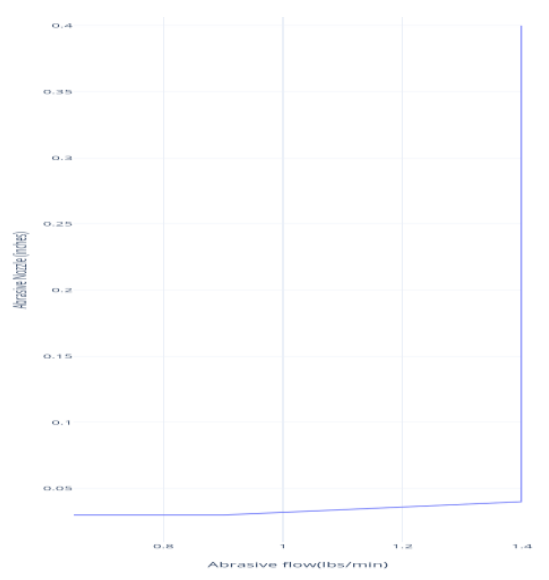

Figure 3 : Abrasive nozzle - Abrasive flow

When nozzle diameter is greater than the energy density becomes lower as compared to the smaller nozzle diameter, deducting the precision and accuracy for machining operations [2].

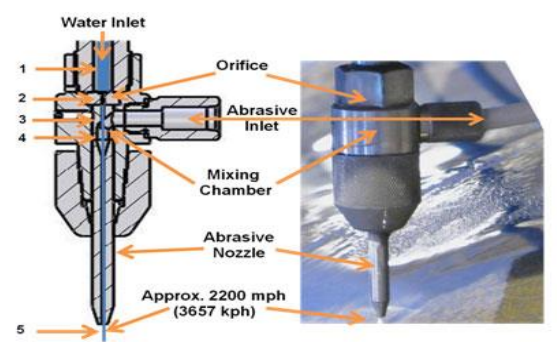

Figure 4 : Nozzle and Orifice

Nozzle is the shortest-lived component in the waterjet machine, which makes it important to analyze wear characteristics of the nozzle. Likewise, causing wear of the nozzle will affect the cutting quality of material and geometry of machining operation. Typical nozzle characteristics are given in the following table for a better selection of nozzle. 


\begin{tabular}{|l|l|}
\multicolumn{1}{|c}{ PARAMETERS_ } & \multicolumn{1}{c|}{ RECOMMENDED_VALUES } \\
\hline Nozzle_length & $50.8 \mathrm{~mm}$ \\
\hline Nozzle_diameter & $1.14 \mathrm{~mm}$ \\
\hline Nozzle_Inlet_angle & $60^{\circ}$ \\
\hline Orifice_diameter & $0.38 \mathrm{~mm}$ \\
\hline Water_pressure & $310 \mathrm{MPa}$ \\
\hline Abrasive_flow & $3.8 \mathrm{~g} / \mathrm{s}$ \\
\hline
\end{tabular}

Figure 5 : Nozzle Characteristics

\section{B. Precision and Quality -}

For the indigenous method for performing abrasive waterjet machining, there are some key important factors which should be taken into consideration to improve efficiency of the cut and to reduce tolerance of the workpiece. The factors which affect precision and quality are as follows -

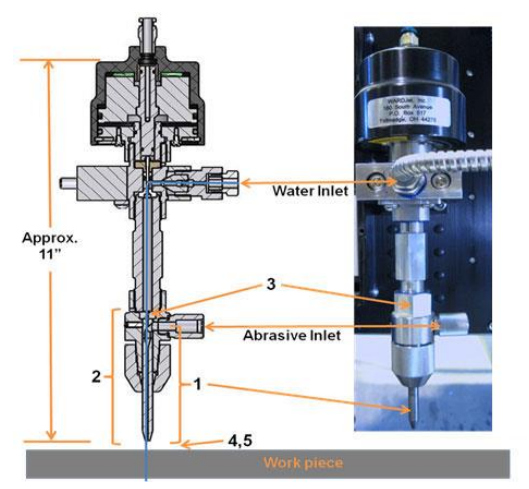

Figure 6 (a) : Schematic of Abrasive Waterjet cutting

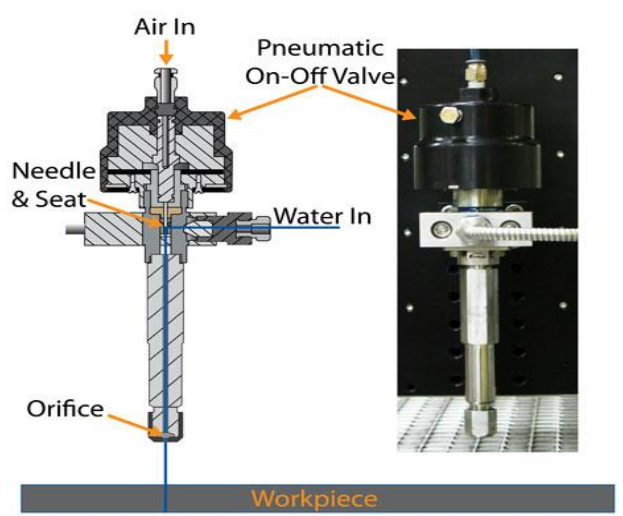

Figure 6 (b) : Schematic of Abrasive waterjet cutting

\section{Water Pressurisation:}

Due to the high-pressure velocity of the jet bestows Venturi Effect or vacuum instantly after a mixing chamber located beneath the orifice. Cutting speed of the abrasive waterjet can be increased when the abrasive reaches the saturation point with a subsequent decrease in speed [6]. When the abrasive exceeds the saturation level then the tube gets choked. This extreme speed and power erode the cutting material [8]. The water pressure is only for providing an efficient amount of speed and power, substantially abrasive transmits the cutting ability of waterjet machining.

\section{Nozzle Height:}

The nozzle height from the workpiece is a significant prospect for machining operations. It provides greater accuracy and precision for cutting operations [9] syazwani et. al. (2016). The optimum distance between the workpiece and the nozzle should be 1.5 $\mathrm{mm}$ to $3.00 \mathrm{~mm}$ to obtain an accurate cut. As the height increases, there is a partial cut on the workpiece which leads to wider kerf angle arising inaccuracies of cut.

\section{Kerf Angle and speed :}

Kerf angle can be stated as the dimensional difference between the top cut and the bottom cut on the workpiece. If the speed is too fast the initial top cut is wider as compared to the bottom cut of the workpiece. Similarly, there are dimensional inaccuracies if appropriate instructions wouldn't be followed.

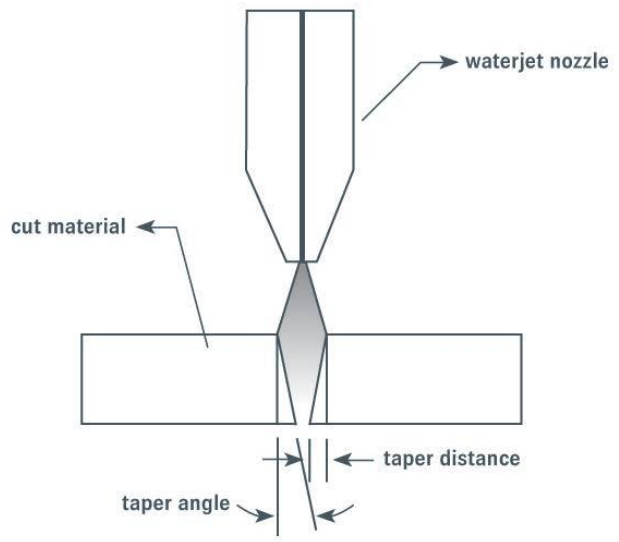

Figure 7: Kerf angle

These factors matter the most for execution of proper and precise machining operations.

C. Comparison of Abrasive Waterjet cutting with other methods.

\begin{tabular}{|l|l|l|l|}
\hline $\begin{array}{l}\text { Method of } \\
\text { cutting }\end{array}$ & $\begin{array}{l}\text { Abrasive } \\
\text { WaterJets }\end{array}$ & Laser Beam & $\begin{array}{l}\text { Plasma } \\
\text { Bram }\end{array}$ \\
\hline Speed & Slow & Fast & Fast \\
\hline Material & Thick and & Thin and & Medium and \\
\hline
\end{tabular}


International Journal of Engineering Applied Sciences and Technology, 2020

Vol. 4, Issue 11, ISSN No. 2455-2143, Pages 471-473

Published Online March 2020 in IJEAST (http://www.ijeast.com)

\begin{tabular}{|l|l|l|l|}
\hline thickness & thin & medium & thick \\
\hline $\begin{array}{l}\text { Thermal } \\
\text { Deformation }\end{array}$ & Lack & Yes & Yes \\
\hline Composites & Yes & No & No \\
\hline $\begin{array}{l}\text { Material } \\
\text { Hardening }\end{array}$ & No & Yes & Yes \\
\hline $\begin{array}{l}\text { Hazardous } \\
\text { Vapours }\end{array}$ & No & Yes & Yes \\
\hline $\begin{array}{l}\text { Multi-layer } \\
\text { cutting }\end{array}$ & Possible & Impossible & Impossible \\
\hline
\end{tabular}

[4] Krajcarz et. al. (2014).

\section{CONCLUSION}

Abrasive waterjet machining analysis provided us an applicable method for determining precision and nozzle characteristics required for it. The comparison between other techniques improved our perspective for a better selection of nozzle, abrasive materials with precision and accuracy of machining operations. Waterjet Cutting doesn't cause thermal Deformation and Material hardening while performing Operations which makes this technique most effective.

\section{REFERENCES}

1. Khan, A. A., \& Haque, M. M. (2007). Performance of different abrasive materials during abrasive water jet machining of glass. Journal of materials processing technology, 191(1-3), 404-407.

2. Nanduri, M., Taggart, D. G., \& Kim, T. J. (2000). A study of nozzle wear in abrasive entrained water jetting environment. J. Trib., 122(2), 465-471.

3. Xu, S., \& Wang, J. (2006). A study of abrasive waterjet cutting of alumina ceramics with controlled nozzle oscillation. The International Journal of Advanced Manufacturing Technology, 27(7-8), 693.

4. Krajcarz, D. (2014). Comparison metal water jet cutting with laser and plasma cutting. Procedia Engineering, 69(1), 838-843.

5. Wang, J. (2007). Predictive depth of jet penetration models for abrasive waterjet cutting of alumina ceramics. International Journal of Mechanical Sciences, 49(3), 306-316.

6. Hashish, M. (1988). Visualization of the abrasivewaterjet cutting process. Experimental mechanics, 28(2), 159-169.

7. Terzis, A. J., Nowak, G., Rentzsch, O., Arnold, H., Diebold, J., \& Baretton, G. (1989). A new system for 